\title{
HARMONIC MEASURE AND RADIAL PROJECTION
}

\author{
DONALD E. MARSHALL AND CARL SUNDBERG
}

\begin{abstract}
Among all curves in the closed unit disk that meet every radius, there is one, $\gamma_{0}$, whose harmonic measure at the origin is minimal. We give an explicit description of $\gamma_{0}$ and compute its harmonic measure. We also give a quadratically convergent algorithm to compute the harmonic measure of one side of a rectangle at its center.
\end{abstract}

\section{INTRODUCTION}

Let $W$ be a domain in the plane and let $E$ be a Borel subset of the boundary of $W, \partial W$. The harmonic measure of $E$ at $z \in W$ (relative to $W$ ) is the solution to the Dirichlet problem in $W$ with boundary values 1 on $E$ and 0 on $\partial W \backslash E$. More precisely, let $\chi_{E}(\varphi)=1$ for $\varphi \in E, \chi_{E}(\varphi)=0$ for $\varphi \in \partial W \backslash E$. Then the harmonic measure at $z$ is

$$
w(z, E, W)=\sup \{u(z): u \text { is subharmonic in } W \text { and }
$$

$$
\left.\limsup _{z \rightarrow \varphi} u(z) \leq \chi_{E}(\varphi) \text { for } \varphi \in \partial W\right\} .
$$

If $F$ is a Borel subset of the closure of $W$, the harmonic measure of $F$ at $z$ will mean the harmonic measure of $F \cap \partial(W \backslash F)$ with respect to the component of $W \backslash F$ containing $z$. See Ahlfors [1] for an introduction to this subject.

Harmonic measure is extremely useful for estimating the growth of analytic and harmonic functions, see Garnett [6]. An early example is the CarlemanMilloux problem [4,12,13]: Suppose that $f$ is analytic and $|f(z)| \leq M$ in the unit disk $D$. Suppose further that $|f(z)| \leq m$ on a curve $\gamma$ that connects the origin to $\partial D$. How large can $\left|f\left(z_{0}\right)\right|$ be at a given point $z_{0}$ ? By the two constant theorem [1], $\left|f\left(z_{0}\right)\right| \leq m^{w} M^{1-w}$ where $w=w\left(z_{0}, \gamma, D \backslash \gamma\right)$. (Milloux [12] attributes a version of this fact to Carleman.) What is needed, therefore, is a lower bound for $w$ which depends only on the fact that $\gamma$ connects 0 to $\partial D$. A more general version of this problem was solved independently by Beurling

Received by the editors January 5, 1988.

1980 Mathematics Subject Classification (1985 Revision). Primary 30C85, 31A15; Secondary 30C30, 30C70.

Key words and phrases. Harmonic measure, extremal length.

Research supported in part by NSF Grant DMS 8601467. 
[3] and R. Nevanlinna [14]. Kakutani [9] proved later that $w(z, E, W)$ equals the probability that a Brownian traveler starting from the point $z$ first hits $\partial W$ in the set $E$. Thus the "further away" the set $E$ is from $z$, the smaller its harmonic measure. Beurling's result fits this intuitive notion. It says that if $z_{0}>0$, then $w\left(z_{0}, \gamma, D \backslash \gamma\right)$ is minimal when $\gamma$ is the radius $[-1,0]$.

We wish to consider a variant of this problem, namely: Suppose that $f$ is analytic and $|f(z)| \leq M$ in $D$. Suppose further that $|f(z)| \leq m$ on a curve $\gamma$ that meets every radius. In other words, for each $\theta, 0 \leq \theta \leq 2 \pi$, there is a point $r e^{i \theta} \in \gamma, 0<r<1$. How large can $|f(0)|$ be? Again, by the two constant theorem, $|f(0)| \leq m^{w \prime} M^{1-w}$ where $w=w(0, \gamma, D \backslash \gamma)$. If $\gamma$ is a closed curve, then by the maximum principle, $|f(z)| \leq m$, i.e., $w=1$. What is needed, again, is a lower bound for $w(0, \gamma, D \backslash \gamma)$.

Theorem 1. Suppose $\gamma$ is a continuum in the closed unit disk $\bar{D}$ that meets every radius. Then the harmonic measure at the origin for $\gamma$ in $D \backslash \gamma$ is at least $c_{0}=.977126698498665669 \ldots$. This lower bound is achieved only for rotations and reflections of the curve $\gamma_{0}$ given by $\gamma_{0}=\gamma^{1} \cup \gamma^{2}$ where $\gamma^{1}$ is the lower half of the unit circle, $\{z:|z|=1, \operatorname{Im}(z) \leq 0\}$, and $\gamma^{2}$ is the image of the half-hyperbola $x^{2} / 3-y^{2}=1 / 4, y \leq 0, x>0$, under the linear fractional transformation $(1-z) /(1+z), z=x+i y$ (see Figure 3 ). The constant $c_{0}$ is equal to the harmonic measure at the center of a $1: 3$ rectangle for the two long sides.

A version of Hall's lemma [10] states that if $E \subset D$ and if $E^{*}=\left\{e^{i \theta}: r e^{i \theta} \in\right.$ $E$, some $r>0\}$ is the radial projection of $E$ on $\partial D$, then $w(0, E, D \backslash E) \geq$ $c w\left(0, E^{*}, D\right)=c\left|E^{*}\right| / 2 \pi$, where $\left|E^{*}\right|$ is the Lebesgue measure of $E^{*}$. Unlike Beurling's theorem, $c \neq 1$. Fuchs $[2$, p. 493] has asked what the optimal constant $c$ is. Our result shows how close $c$ is to 1 in a special case.

In $\S 2$, we use extremal length to determine the optimal curve $\gamma_{0}$. In $\S 3$, we give a more explicit description of $\gamma_{0}$ and give a quadratically convergent algorithm to compute $c_{0}$. In a future paper, with different techniques, we will treat the more general problem where the curve $\gamma$ meets radii $\left\{r e^{i \theta}: 0<r<1\right\}$ with $0 \leq \theta \leq \alpha<2 \pi$. In [5] it is proved that if $\alpha \leq \pi$ then the minimal harmonic measure is $\alpha / 2 \pi$. These authors pose the problem of determining the maximal $\alpha$ for which this remains true. Our analysis will yield a computation of this extremal value. In $\S 4$, we show that the constant $c_{0}$ in Theorem 1 is not the optimal constant $c$ in the problem mentioned in the previous paragraph. In the process of showing $c<c_{0}$, we give an alternate proof of a result of Hayman [7].

We would like to mention that numerical computations were indispensable at various stages of this project, though the proof of Theorem 1 does not depend upon them. The extremal curve [Figure 3] was drawn using the conformal mapping technique given in Marshall and Morrow [11]. We would like to thank J. Morrow for his assistance. It was only after viewing this picture that we 
discovered the simple formula for it given in $\S 3$. It was because that mapping technique is so well suited to map regions $D \backslash \gamma$ to $D$, that L. Carleson passed this problem on to us, for which we would like to thank him.

\section{Proof of Theorem 1}

In the course of the proof of Theorem 1, we will need the notion of extremal length. If $F$ is a family of locally rectifiable arcs in a region $U$ and if $\rho$ is a nonnegative Borel measurable function on $U$ (such a function will henceforth be called a metric), we define the $\rho$-length of $\phi \in F$ to be

$$
L(\phi, \rho)=\int_{\phi} \rho|d z|
$$

and the $\rho$-area of $U$ to be

$$
A(U, \rho)=\int_{U} \rho^{2} d A
$$

where $d A$ is the Lebesgue measure on $U$. The extremal length of $F$ in $U$ is defined to be

$$
\lambda_{U}(F)=\sup _{\rho} \inf _{\phi \in F}\left\{\frac{L(\phi, \rho)^{2}}{A(U, \rho)}\right\} .
$$

See Ahlfors [1] for an introduction to extremal length. We will use only three elementary facts about extremal length. The first is that it is conformally invariant, i.e., if $f$ is a conformal map of $U$ onto an open set $U^{\prime}$ and if $F^{\prime}$ is the image of $F$ then $\lambda_{U}(F)=\lambda_{U^{\prime \prime}}\left(F^{\prime}\right)$. Indeed, if $w=f(z)$, and if $\rho$ is a metric on $U^{\prime}$ then $\rho(w)|d w|$ is transformed to the metric $\rho(f(z))\left|f^{\prime}(z)\right||d z|$ on $U$. The second needed fact is a beautiful criterion due to Beurling; see [1] for the extremality of a metric.

Theorem 2 (Beurling). A metric $\rho_{0}$ is extremal for $F$ if $F$ contains a subfamily $F_{0}$ with the following properties:

$$
\int_{\varphi} \rho_{0}|d z|=\inf _{\phi \in F} L\left(\phi, \rho_{0}\right) \quad \text { for all } \varphi \in F_{0} .
$$

(2) For real-valued $h$ in $U$ : if $\int_{\varphi} h|d z| \geq 0$ for all $\varphi \in F_{0}$ then $\int_{U} h \rho_{0} d A \geq 0$.

Moreover, in this case, the metric $\rho_{0}$ is (a.e. $d A$ ) the unique extremal metric, up to multiplication by a positive constant.

The major difficulty in extremal length problems is to discover the extremal metric. Once such a metric is found, Beurling's criterion is usually used to prove it is extremal. We suggest the reader use Beurling's criterion to prove that $\rho \equiv 1$ is the extremal metric for curves that connect opposite sides of a rectangle.

Since the uniqueness portion of this theorem is not explicitly stated in [1], we shall include the proof for completeness. 
Let $\rho$ be a metric normalized by

$$
\inf _{\varphi \in F} L(\varphi, \rho)=\inf _{\varphi \in F} L\left(\varphi, \rho_{0}\right) .
$$

Then

$$
\int_{\varphi} \rho|d z| \geq \int_{\varphi} \rho_{0}|d z| \quad \text { for all } \varphi \in F_{0} .
$$

Let $h=\rho-\rho_{0}$. By (2)

$$
\int_{U} \rho_{0}^{2} d A \leq \int_{U} \rho_{0} \rho d A
$$

By the Cauchy-Schwarz inequality

$$
\int_{U} \rho_{0}^{2} d A \leq \int_{U} \rho^{2} d A
$$

This proves that $\rho_{0}$ is extremal. If $\int_{U} \rho^{2} d A=\int_{U} \rho_{0}^{2} d A$, then

$$
\int_{U}\left(\rho_{0}-\rho\right)^{2} d A=2 \int_{U}\left(\rho_{0}^{2}-\rho_{0} \rho\right) d A \leq 0 .
$$

Hence $\rho_{0}=\rho$ a.e. $d A$.

The third fact needed in the proof is given in the following elementary lemma.

Lemma 3. Suppose $T$ is a one-to-one, conformal map (either analytic or antianalytic) from $U$ onto $U$ such that $T \circ T(z)=z$ on $U$. Suppose further that the curve family $F$ satisfies $T(F)=F$. To compute the extremal length of the family $F$, it suffices to consider metrics $\rho$ with $\rho \circ T\left|T^{\prime}\right|=\rho$.

Proof. Let $\tilde{\rho}=\rho \circ T\left|T^{\prime}\right|$ and let $\rho_{1}=(\rho+\tilde{\rho}) / 2$. Note that

$$
\int_{U^{\prime}} \rho^{2} d A=\int_{U} \tilde{\rho}^{2} d A \text { and } \int_{T(\varphi)} \rho|d z|=\int_{\varphi} \tilde{\rho}|d z| .
$$

This implies that

$$
\int_{U^{U}} \rho_{1}^{2} d A=\int_{U^{\prime}} \rho^{2} / 2 d A+\int \rho \tilde{\rho} / 2 d A \leq \int_{U} \rho^{2} d A
$$

and

$$
\int_{\varphi} \rho_{1}|d z| \geq \min \left[\int_{\varphi} \rho|d z|, \int_{T(\varphi)} \rho|d z|\right] .
$$

Thus $\inf L\left(\varphi, \rho_{1}\right)^{2} / A\left(U, \rho_{1}\right) \geq \inf L(\varphi, \rho)^{2} / A(U, \rho)$. Since $T \circ T(z)=z$, we conclude that $\rho_{1} \circ T\left|T^{\prime}\right|=\rho_{1}$.

We will now prove Theorem 1. To obtain a lower bound for $w(0, \gamma, D \backslash \gamma)$, we may suppose, by an approximation, that $\gamma$ is a piecewise smooth Jordan arc. We will later show there is an extremal curve $\gamma_{0}$ which is piecewise smooth. Indeed, if $f$ is the conformal map of the component of $D \backslash \gamma$ containing 0 (which is necessarily simply connected) onto the disk $D$, then there is an arc $J \subset \partial D$ such that $f^{-1}(z)$ tends to $\gamma$ and $z$ approaches the interior of $J$ and $f^{-1}(z)$ approaches $\partial D$ as $z$ approaches the interior of $\partial D \backslash J$. Let $\Gamma=\{z \in$ 
$D: w(z, J, D)=1-\varepsilon\}$, and let $\gamma_{1}=f^{-1}(\Gamma)$. Clearly $\gamma_{1}$ is smooth, meets each radius and $w\left(0, \gamma_{1}, D \backslash \gamma_{1}\right)=w(0, \gamma, D \backslash \gamma) /(1-\varepsilon)$. By altering $\Gamma$ slightly near the ends of $J$, and rotating $D$, we may suppose that $\gamma$ is a smooth map from the interval $[0,1]$ into $\bar{D}$ with $\gamma(0)>0$. By taking a subarc of $\gamma$, if necessary, we may suppose that $\gamma$ intersects the interval $(0,1]$ in exactly two points: $\gamma(0)$ and $\gamma(1)$. By reparameterizing $\gamma$, we may suppose $\gamma(0)>\gamma(1)$.

There is another reduction, which comes from [5]. The proof can be completed without it, however it makes the proof a little easier. Let $r=\min \{a \in$ $[-1,0): a=\gamma(t)$ for some $t\}$, let $E=[-1, r] \cup[\gamma(0), 1]$, and let $W=D \backslash E$. Finally, let $k$ be the conformal map of $D \backslash E$ onto $D$ with $k(0)=0$ and $k$ real-valued on $(-1,1)$. Clearly,

$$
w(0, k(\gamma), D \backslash k(\gamma))=w(0, \gamma, D /(\gamma \cup E)) \leq w(0, \gamma, D \backslash \gamma) .
$$

Note that $k(\gamma)$ meets every radius, and meets $\partial D$ in at least two points. If $\gamma\left(t_{0}\right) \in \partial D$ and $\gamma\left(t_{1}\right) \in \partial D, t_{0}<t_{1}$, then we may replace $\gamma$ on the interval $\left[t_{0}, t_{1}\right]$ by an arc on $\partial D$ from $\gamma\left(t_{0}\right)$ to $\gamma\left(t_{1}\right)$, resulting in smaller harmonic measure, by the maximum principle. The resulting curve will still meet every radius since $\gamma$ begins and ends on the interval $(0,1]$. Replacing $\gamma$ with $\bar{\gamma}=$ $\{\bar{z}: z \in \gamma\}$, if necessary, we may suppose that $\operatorname{Im}(\gamma(t))<0$ for $t<\varepsilon$ and $\operatorname{Im}(\gamma(t))>0$ for $t>1-\varepsilon$. In other words, we may suppose that the curve $\gamma$ begins at 1 , follows the unit circle clockwise at least as far as the point -1 , ends at $\gamma(1)>0$, meets each radius, and crosses the interval $(-1,1)$ between -1 and 0 (if at all). (See Figure 1.)

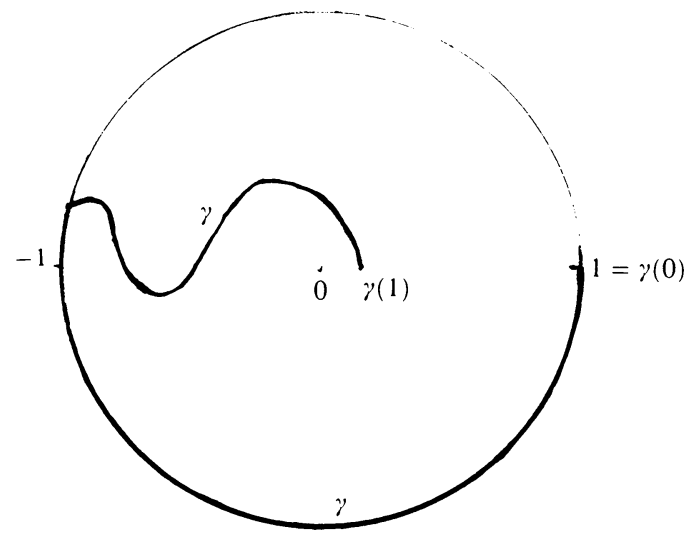

FIGURE 1

Let $f$ be the conformal map of $D \backslash \gamma$ onto $D$, with $f(0)=0$, where $f(\partial D \backslash \gamma)$ consists of an arc $\alpha$ on $\partial D$ centered at 1 . By the conformal invariance of harmonic measure, and the Poisson integral formula,

$$
w(0, \gamma, D \backslash \gamma)=1-|\alpha| / 2 \pi
$$

where $|\alpha|$ denotes the length of the arc $\alpha$. So to minimize $w(0, \gamma, D \backslash \gamma)$, we must maximize $|\alpha|$. Let $F$ denote the family of rectifiable arcs $\phi$, defined for 
$0 \leq t \leq 1$, with $\phi(0) \in \alpha, \phi(1) \in \alpha, \phi(t) \in D \backslash\{0\}$ for $0<t<1$ and such that 0 and -1 are not in the same component of $\bar{D} \backslash(\phi \cup \alpha)$. In other words, each $\phi$ encloses 0 and begins and ends on $\alpha$. Clearly if we increase $\alpha$, we will increase the family $F$ and hence decrease the corresponding extremal length. Let $G_{\gamma}$, denote the family $\left\{f^{-1}(\phi): \phi \in F\right\}$. These are curves that separate 0 from $\gamma$ and begin and end on $\partial D \backslash \gamma$. By the conformal invariance of $\lambda$, our problem is to find the curve that minimizes $\lambda_{D \backslash \gamma}\left(G_{\gamma}\right)$.

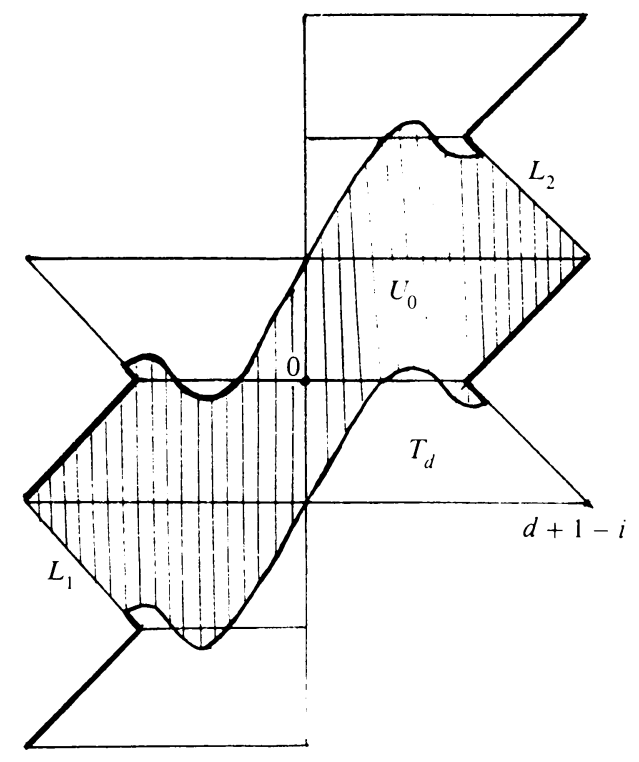

FIGURE 2

Let $T_{d}$ be the trapezoid with vertices $v_{1}=0, v_{2}=d>0, v_{3}=d+1-i$, and $v_{4}=-i$. There is a unique number $d>0$ and corresponding conformal map $h_{d}$ with maps $T_{d}$ onto the upper half disk $D^{+}=\{z:|z|<1$ and $\operatorname{Im}(z)>0\}$ with $h\left(v_{1}\right)=0, h\left(v_{2}\right)=-1, h\left(v_{3}\right)=+1$ and $h\left(v_{4}\right)=\gamma(1)$. Let $S_{1}$ be the union of $T_{d}$ together with its reflection about the line $\operatorname{Im}(z)=0$. Let $S_{2}$ be the reflection of $S_{1}$ about the $\operatorname{line} \operatorname{Im}(z)=1$, let $S_{3}$ be the reflection of $S_{1}$ about the line $\operatorname{Re}(z)=0$ and let $S_{4}$ be the reflection of $S_{3}$ about the line $\operatorname{Im}(z)=-1$. Finally, let $S=S_{1} \cup S_{2} \cup S_{3} \cup S_{4}$ (see Figure 2). The region $S$ is the union of 8 trapezoids congruent to $T_{d}$. The map $h_{d}$ extends analytically to $S$ by the Schwarz reflection principle. Let $U_{0}$ be the component of $\operatorname{int}(S) \backslash\{z \in S: h(z) \in \gamma\}$ containing the origin, where $\operatorname{int}(S)$ is the interior of $S$. Since $\gamma$ crosses $(-1,1)$ only between -1 and 0 , the boundary of $U_{0}$ consists of the two curves $\{z \in S: h(z) \in \gamma\}$ together with two line segments $L_{1}$ and $L_{2}$ with $L_{1} \in \partial S_{4}$ and $L_{2} \in \partial S_{2}$, each with slope -1 . On the region $\bar{U}_{0}$, the map $h$ is a two-to-one cover of $D \backslash \gamma$. To see this, note that $h$ is twoto-one on $S_{1} \cup S_{3}$, and that if $z \in S$ and $z \pm 2 i \in S$, then $h(z)=h(z \pm 2 i)$. Moreover, if $z \in U_{0}$, exactly one of the points $z, z+2 i, z-2 i$ is in $S_{1} \cup S_{3}$, call it $u(z)$. The function $u$ extends to a one-to-one map of $\bar{U}_{0}$ onto $S_{1} \cup S_{3}$. 
Thus $h$ is two-to-one; in fact, $h(-z)=h(z)$. Thus for each $\phi \in F$, there is a rectifiable arc $\psi$ in $U_{0}$ with endpoints on $L_{1} \cup L_{2}$ that either "encloses" the origin or else connects $L_{1}$ and $L_{2}$. Let $H_{d}$ be the family in $U_{0}$ of all such curves. To compute the length of the family $H_{d}$, by Lemma 3, we need only consider metrics $\rho$ on $U_{0}$ with $\rho(-z)=\rho(z)$. Any such metric can be written in the form $\rho(z)=q\left(h_{d}(z)\right)\left|h_{d}^{\prime}(z)\right|$ where $q$ is a metric on $D \backslash \gamma$. Clearly $A\left(U_{0}, \rho\right)=2 A\left(U_{0}, q\right)$ and $L(\psi, \rho)=L\left(h_{d}(\psi), q\right)$, so that $\lambda_{U_{0}}\left(H_{d}\right)=$ $\lambda_{D \backslash \gamma}\left(G_{y}\right) / 2$. Thus we wish to minimize $\lambda_{U_{0}}\left(H_{d}\right)$.

Let $\rho \equiv 1$ on $U_{0}$. The $\rho$-area of $T_{d}$ is $d+1 / 2$, so by use of the above map $u, \int_{U_{0}} \rho d A=4(d+1 / 2)$. Note that the distance from $L_{1}$ to the line through the origin with slope -1 equals $\sqrt{2}+d / \sqrt{2}$. Thus every curve $\psi \in H_{d}$ has length

$$
\int_{\psi}|d z| \geq 2\left(\sqrt{2}+\frac{d}{\sqrt{2}}\right)
$$

We conclude

$$
\lambda_{U_{0}}\left(H_{d}\right) \geq \frac{[2(\sqrt{2}+d / \sqrt{2})]^{2}}{4(d+1 / 2)} \geq 3 .
$$

The latter inequality is strict, unless $d=1$. Now let $d=1$ and let $\sigma$ be the straight line from $v_{2}$ to $v_{4}$. Let $\tau$ be the bottom half of the unit circle $=\{z:|z|=1$ and $\operatorname{Im}(z) \leq 0\}$. Let $\gamma_{0}=\tau \cup h(\sigma)$ (see Figure 3). The corresponding $U_{0}$ is then clearly a 3 by 1 rectangle whose sides have slope +1 and -1 (see Figure 4). Let $H_{0}$ consist of the line segments of slope 1 connecting opposite sides of $U_{0}$. By Beurling's criterion, $\rho_{0} \equiv 1$ on $U_{0}$ is the unique extremal metric for this family and hence $\lambda_{U_{0}}\left(H_{0}\right)=3$. This proves that $\min \lambda_{D \backslash \gamma}\left(G_{\gamma}\right)=6$ and that $\gamma_{0}$ is extremal. Moreover, any other extremal curve would necessarily have $d=1$. The proof also shows that the Euclidean metric on $U_{0}$ must be extremal. By Beurling's criterion, the extremal metric always comes from the conformal map of $U_{0}$ onto a rectangle. Hence $U_{0}$ must be a rectangle in the extremal case.

So far, we have supposed that the candidates for the extremal curve are piecewise smooth. We would now like to prove that $\gamma_{0}$ is the only continuum in $\bar{D}$, meeting every radius, for which the harmonic measure is minimal. Let $\gamma$ be such a continuum. Then $\gamma$ may be approximated, as indicated above, by curves $\gamma_{n}, n=1,2, \ldots$, with corresponding regions $U_{0}\left(\gamma_{n}\right)$ and conformal maps $h_{n}$ of $U_{0}\left(\gamma_{n}\right)$ onto $D \backslash \gamma_{n}$. Let $k_{n}$ be the conformal map of $U_{0}\left(\gamma_{n}\right)$ onto a rectangle $R_{n}$ so that $\rho_{n}=\left|k_{n}^{\prime}\right|$ is the extremal metric for $\lambda_{U_{0}\left(\gamma_{n}\right)}\left(H_{d_{n}}\right)$. Since $\lambda_{U_{0}\left(\gamma_{n}\right)}\left(H_{d_{n}}\right)=3$, we may choose a normalization for $k_{n}$ so that $R_{n}$ converges to the 3 by 1 rectangle $U_{0}\left(\gamma_{0}\right)$. Thus there is a subsequence $\left\{k_{n_{j}}^{-1}\right\}$ of $\left\{k_{n}\right\}$ converging uniformly on compact subsets of $U_{0}\left(\gamma_{0}\right)$ to the map $k(z)=z$. We conclude that the corresponding maps $h_{n_{j}}$ must converge uniformly on compact subsets of $U_{0}$ to the map $h_{0}$ of $U_{0}$ onto $D \backslash \gamma_{0}$. Thus $\gamma_{0}$ is the unique extremal continuum. We note that the curve in Figure 3 beginning at 


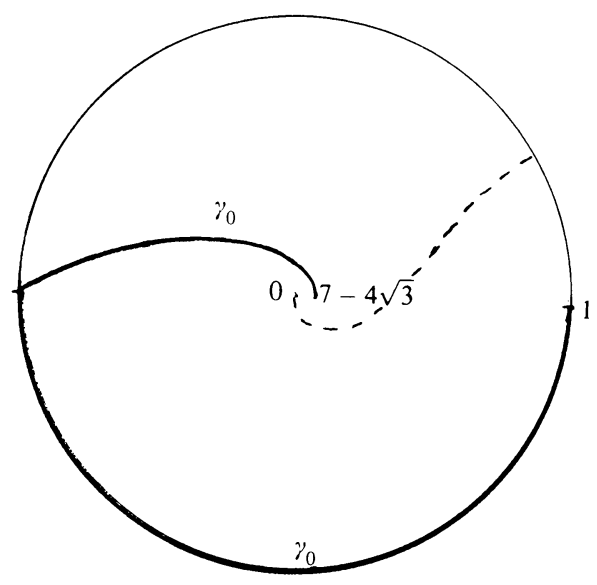

FIGURE 3

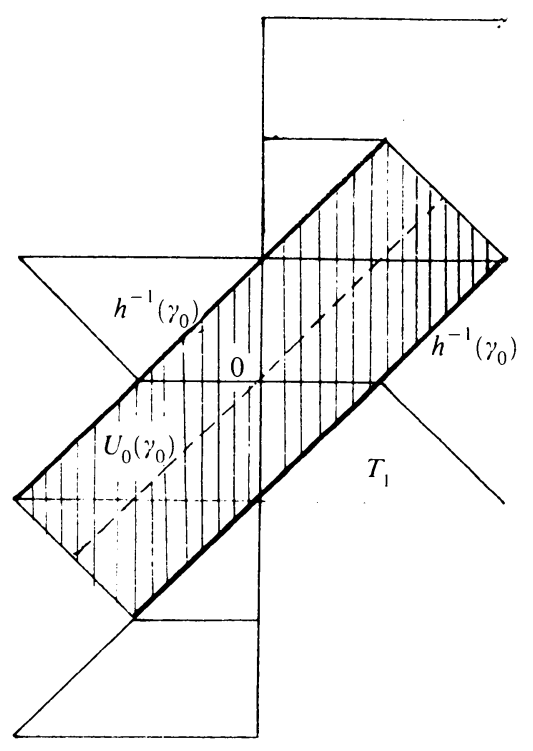

FIGURE 4

the origin and extending to $\partial D$ is, in some sense, the most efficient path from the origin to $\partial D \backslash \gamma$. It is the image under the map $h$ of the straight line in $U_{0}$ through 0 and with slope 1 .

We remark here that our discovery of the extremal metric was motivated by the deep work of Jenkins [8]. It is not hard to show that Jenkins' theorem implies that the extremal curve (suitably normalized) must consist of an arc from 1 to a point $e^{i \theta}$ on $|z|=1$ then an $\operatorname{arc}$ in $D$ from $e^{i \theta}$ to a point $x \in D$, 
$x>0$. Moreover, the extremal metric is given by

$$
\rho^{2}=c \frac{\left(z-e^{i \theta}\right)}{z(z-1)(z-x)(z-1 / x)}
$$

where $c$ is a constant. Jenkins show that for each $x$ there is a unique $\theta$ so that this metric corresponds to the curve, of minimal harmonic measure, that ends at $x$ and meets every radius. He does not have a formula for $\theta$ in terms of $x$. The difficulty is in deciding which $x$ corresponds to the extreme case in our problem. It may be instructive to note that the trapezoids $T_{d}$ that we constructed do not correspond to these metrics, unless $d=1$. Indeed, one can show that $e^{i \theta} \neq-1$ if $x$ is not the endpoint of our external $\gamma_{0}$, yet the metrics associated with the rectangles formed from $T_{d}$ will have a singularity at -1 . We do not know of any "nice" conformal maps corresponding to the metrics of Jenkins. We will reexamine Jenkins' metrics in another paper, as mentioned in the introduction.

\section{The eXtremal CURVE}

Let $\gamma_{0}$ be the extremal curve in $\S 2$, let $h$ be the associated map of the rectangle $U_{0}$ onto $D \backslash \gamma_{0}$, and let $f$ be the conformal map of $D \backslash \gamma_{0}$ onto $D$ with $f(0)=0$, and with $f(\partial D)$ equal to an arc $\alpha$ on $\partial D$ centered at 1 . If $\varphi_{1}$ is a conformal map of $D$ onto $U_{0}$ with $f \circ h \circ \varphi_{1}(0)=0$ then $\phi=f \circ h \circ \varphi_{1}$ is a two-to-one map of $D$ onto $D$ with $\phi(0)=\phi^{\prime}(0)=0$. An elementary argument shows that $\phi(z)=c z^{2}$ where $c$ is a constant. Hence $\sqrt{f \circ h}$ is a conformal map of the rectangle $U_{0}$ onto $D$, which maps the four vertices to points $\beta, \bar{\beta},-\beta, \bar{\beta}$. Note that the endpoints of the arc $\alpha$ are $\beta^{2}$ and $\bar{\beta}^{2}$, and hence $w\left(0, \gamma_{0}, D \backslash \gamma_{0}\right)=1-2|\theta| / \pi$ where $\beta=e^{i \theta}$. Note that $U_{0}$ has side ratio $3: 1$. The quantity $|\theta| / \pi$ is the harmonic measure, at the center, of one of the short sides of a $3: 1$ rectangle. By the Schwarz-Christoffel formula, the number $\theta$ is the solution to the integral equation $(0<\theta<\pi / 4)$ :

$$
3 \int_{0}^{\theta}\left[\frac{1}{\cos 2 s-\cos 2 \theta}\right]^{1 / 2} d s=\int_{\theta}^{\pi / 2}\left[\frac{1}{\cos 2 \theta-\cos 2 s}\right]^{1 / 2} d s .
$$

The solution of this equation by standard methods of numerical integration, while possible, is not trivial and converges slowly. Trefethen [15] gives a rapidly converging algorithm to compute $\theta$ based on a different method. An algorithm to compute $\theta$ seems to be of some numerical interest. For example, Trefethen [16] has shown the use of $\theta$ in the design of a resistor. This is equivalent to finding the parameter $k, 0 \leq k \leq 1$, for which the Jacobi elliptic function $s n(z, k)$ maps a 1 by 3 rectangle onto the upper half-plane. We give here a method that is very simple and converges even faster (quadratic convergence) than Trefethen's method. It is based on the following observation. Suppose $R_{L}$ is a rectangle centered at the origin with sides parallel to the axes, with height 1 and length $L \geq 1$. Let $S_{L}$ be the semi-infinite strip $\{z: \operatorname{Re}(z)>$ $-L / 2,|\operatorname{Im}(z)|<1 / 2\}$. Let $w_{R_{L}}(z)$ be the harmonic measure of the left-hand 
edge of $R_{L},\left\{z \in \bar{R}_{L}: \operatorname{Re}(z)=-L / 2\right\}$, at the point $z$ and let $w_{S_{L}}(z)$ be the harmonic measure of the left edge of $S_{L},\left\{z \in \bar{S}_{L}: \operatorname{Re}(z)=-L / 2\right\}$, at the point $z$. Clearly, $w_{R_{L}}(z) \leq w_{S_{L}}(z)$ by the maximum principle. On $\operatorname{Re}(z)=$ $L / 2, w_{S_{L}}(z) \leq w_{S_{L}}(L / 2)$. Thus for $z \in \partial R_{L}$

$$
w_{S_{L}}(z)+w_{S_{L}}(-z) \leq\left\{1+w_{S_{L}}(L / 2)\right\}\left\{w_{R_{L}}(z)+w_{R_{L}}(-z)\right\} .
$$

By the maximum principle, this inequality holds at $z=0$. We conclude that

$$
\frac{w_{S_{L}}(0)}{1+w_{S_{L}}(L / 2)} \leq w_{R_{L}}(0) \leq w_{S_{L}}(0)
$$

Note that $w_{S_{L}}(L / 2)=w_{S_{2 L}}(0)$. By explicitly mapping $S_{L}$ onto the upper-half plane and using elementary trigonometric identities, it is easy to show that

$$
w_{S_{L}}(0)=\frac{4}{\pi} \tan ^{-1}\left(e^{-\pi L / 2}\right) .
$$

Hence

$$
\frac{\frac{4}{\pi} \tan ^{-1}\left(e^{-\pi L / 2}\right)}{1+\frac{4}{\pi} \tan ^{-1}\left(e^{-\pi L}\right)} \leq w_{R_{L}}(0) \leq \frac{4}{\pi} \tan ^{-1}\left(e^{-\pi L / 2}\right)
$$

Note that (2) says $w_{R_{L}}(0) \sim \frac{4}{\pi} e^{-\pi L / 2}$ as $L$ tends to $\infty$. We can improve this estimate in the following way. The quantities $w_{R_{L}}(0)$ and $w_{R_{2 L}}(0)$ are related as follows: Let $\varphi_{L}$ be the conformal map of $R_{L}$ onto $D$ so that the images of the four vertices are $e^{i \theta_{L}}, e^{-i \theta_{L}},-e^{i \theta_{L}},-e^{-i \theta_{L}}$, for some number $\theta_{L}, 0<\theta_{L}<\pi / 2$, and $\varphi_{L}^{\prime}(0)>0$. Note that $\varphi_{L}$ is real on $\mathbf{R} \cap R_{L}$ and $\varphi_{L}(0)=0$. Moreover, $w_{R_{L}}(0)=\theta_{L} / \pi$. Note that $\varphi_{L}$ maps the top half of $R_{L}$ onto the upper half disk $D^{+}$. Let $\chi$ be the conformal map of $D^{+}$onto $D$ so that $\chi\left(e^{i \theta_{L}}\right)=\overline{\chi(1)}=-\chi(-1)=-\overline{\chi\left(-e^{-i \theta_{L}}\right)}$. The top half of $R_{L}$ is clearly the image under the map $z \mapsto(z+i / 2) / 2$ of $R_{2 L}$. Hence $\varphi_{2 L}(z)=$ $\chi\left(\varphi_{L}((z+i / 2) / 2)\right)$ and hence $e^{i \theta_{2 L}}=\chi\left(e^{i \theta_{L}}\right)$. An explicit computation of the map $\chi^{-1}$ shows that

$$
\sin \pi w_{R_{L}}(0)=\frac{2 \sqrt{\sin \pi w_{R_{2 L}}(0)}}{1+\sin \pi w_{R_{2 L}}(0)} .
$$

Hence, if we let

$$
x_{n}=\left(\sin \pi w_{R_{2^{n}}}(0)\right)^{-1 / 2}
$$

we have

$$
x_{n-1}^{2}=\frac{1}{2}\left(x_{n}+1 / x_{n}\right) \text {. }
$$

By (1)

$$
0 \leq\left[\frac{1}{w_{R_{2 n L}}(0)}-\frac{1}{w_{S_{2^{n}}}(0)}\right] \leq \frac{w_{S_{2^{n+1}}}(0)}{w_{S_{2^{n} L}}(0)} .
$$

By elementary calculus, if

$$
y=\left(\sin \pi w_{S_{2^{n} L}}(0)\right)^{-1 / 2}
$$


then $0 \leq x_{n}-y \leq c\left(e^{-\pi L / 2}\right)^{2^{n}}$, for some constant $c$. Note that $x_{n}>x_{n-1}>1$ for all $n$, and that if $y>1$,

$$
\left|x_{n-1}-\sqrt{\frac{1}{2}\left(y+\frac{1}{y}\right)}\right| \leq c\left|x_{n}-y\right|
$$

for some constant $c$ ( $c=\frac{1}{4}$ will suffice). Thus if we let $y_{0}=y$ and $y_{k}=$ $\sqrt{\frac{1}{2}\left(y_{k-1}+\frac{1}{y_{k-1}}\right)}, 1 \leq k \leq n$, then by induction

$$
\left|\left(\sin \pi w_{R_{L}}(0)\right)^{-1 / 2}-y_{n}\right| \leq C\left(e^{-\pi L / 2}\right)^{2^{n}}
$$

where $C$ is a constant which can be determined by elementary calculus. In other words, the number of correct digits in the decimal expansion at least doubles when $n$ is increased by 1 . To simplify the algorithm further, note that

$$
y_{0}=\left(\frac{1+e^{-\pi 2^{n} L}}{2}\right) e^{\pi 2^{n-2} L}
$$

and hence

$$
\left|y_{0}-\frac{1}{2} e^{\pi 2^{n-2} L}\right|<\frac{e^{-\pi 2^{n} L}}{2} .
$$

Thus we may replace $y_{0}$ with $\frac{1}{2} e^{\pi 2^{n-2} L}$ and obtain the estimate (3) above with slightly larger constant $C$. Finally

$$
\left|w_{R_{L}}(0)-\frac{1}{\pi} \sin ^{-1}\left(y_{n}^{-2}\right)\right| \leq C\left(e^{-\pi L / 2}\right)^{2^{n}}
$$

for a slightly larger constant $C$. Notice that

$$
\sin ^{-1}\left(y_{n}^{-2}\right)=\tan ^{-1}\left(\frac{2}{y_{n-1}-1 / y_{n-1}}\right) .
$$

Thus we obtain the following quadratically convergent algorithm:

Given $n$ and $L$, let

$$
\begin{aligned}
& y_{0}=\frac{1}{2} e^{\pi L 2^{n-2}}, \\
& y_{k}=\sqrt{\frac{1}{2}\left(y_{k-1}+1 / y_{k-1}\right)}, \quad 1 \leq k \leq n-1,
\end{aligned}
$$

and

$$
w=\frac{1}{\pi} \tan ^{-1}\left(\frac{2}{y_{n-1}-1 / y_{n-1}}\right) .
$$

Then

$$
\left|w_{R_{L}}(0)-w\right| \leq C\left(e^{-\pi L / 2}\right)^{2^{n}} .
$$

For example, with $L=3$ and $n=2$ the computed value for $c_{0}=1-2 w=$ $.977126698498665669 \ldots$ is correct to 18 decimal places. This is virtually a formula for $c_{0}$. We carried out the above estimate more precisely to obtain 
an error less than $10^{-17}$ for any $L \geq 1$, when $n=4$. Of course, for large $L$ one could (and should) reduce the number of steps. The astute reader can use the above technique to give a quadratically convergent algorithm to compute the conformal map of $D$ to any rectangle if he so desires.

The optimal curve $\gamma_{0}$ (see Figure 3) was drawn using the conformal mapping technique given in Marhsall and Morrow [11]. Although the map from the trapezoid $T_{1}$ to the upper half disk cannot be written in terms of elementary conformal maps, the picture of $\gamma_{0}$ led us to believe that there might be a simple formula for $\gamma_{0}$. Recall that $T_{1}$ is the trapezoid with vertices $0,1,2-i$ and $-i$. Let $S_{1}$ be the square with vertices $1,2-i, 1-2 i$ and $-i$. Let $f_{1}$ be the conformal map of $S_{1}$ onto $D$ such that $f_{1}(2-i)=1, f_{1}(1)=i, f_{1}(-i)=$ $-1, f_{1}(1-2 i)=-i$. Then $f_{1}$ maps $S_{1} \cap T_{1}$ onto the upper half disk $D^{+}$ and the right triangle $T_{2}$ with vertices $-i, 1$ and $1-i$ onto the left quarter circle $Q=\{z:|z|<1, \operatorname{Re}(z)<0, \operatorname{Im}(z)>0\}$. Reflecting $T_{2}$ about the line $\operatorname{Re}(z)=\operatorname{Im}(z)$ and $Q$ about the quarter circle on its boundary, we obtain a conformal map of $T_{1}$ onto the union of $D^{+}$and $\{z \in \mathbf{C}: \operatorname{Re}(z)<0, \operatorname{Im}(z)>$ $0\}$. Applying the conformal map $f_{2}=-1 / z^{2}$ to this region, we obtain a conformal map to $\mathbf{C} \backslash \overline{\left(D^{+}\right.} \cup(-\infty,-1]$. Apply the conformal map $f_{3}(z)=$ $(1+z) /(1-z)$ we obtain the region $C \backslash(\{z: \operatorname{Re}(z) \geq 0$ and $\operatorname{Im}(z) \geq 0\} \cup[-1,0]$. Applying the map $f_{4}(z)=(-i z)^{2 / 3}$ we obtain the region $\{z: \operatorname{Im}(z)>0\} \backslash L_{3}$ where $L_{3}$ is a straight line segment from the origin to the point $e^{i \pi / 3}$. The function $g_{1}(z)=3(z-1 / 3)^{1 / 3}(z+2 / 3)^{2 / 3} / 2^{2 / 3}$ maps the upper half plane conformally onto this latter region (see Marshall and Morrow [11] for details). Note that $g_{1}(-1)=-1$. Let

$$
g_{2}(z)=\left(1-4 z^{2}\right) / 3 \text { and } g_{3}(z)=(1-z) /(1+z) .
$$

The upper half-plane is the image of the quarter plane $Q=\{z: \operatorname{Re}(z)>$ $0, \operatorname{Im}(z)<0\}$ under the map $g_{2}$ and $Q=g_{3}\left(D^{+}\right)$. Thus $h=g_{3}^{-1} \circ g_{2}^{-1} \circ$ $g_{1}^{-1} \circ f_{4} \circ f_{3} \circ f_{2} \circ f_{1}$ is the conformal map of $T_{1}$ onto $D^{+}$such that $h(0)=0$, $h(1)=-1$ and $h(2-i)=1$. A direct computation shows that $h(-i)=7-4 \sqrt{3}$. The curve $\gamma_{0} \cap D$ is the image of the straight line segment $L_{4}$ from $-i$ to 1 in $T_{1}$, under the map $h$. To find this curve more explicitly, first note that the image of $L_{4}$ under the map $f_{4} \circ f_{3} \circ f_{2} \circ f_{1}$ is the ray $\left\{r e^{i 2 \pi / 3}: 0<r<\infty\right\}$. To find the image of this curve under the map $g_{2}^{-1} \circ g_{1}^{-1}$, we must find all $z \in Q$ such that $\left\{g_{1} \circ g_{2}(z)\right\}^{3}$ is positive. In other words, $4 z^{2}\left(1-\frac{4}{3} z^{2}\right)^{2}<0$. Taking a square root, we want $\operatorname{Re}\left(4 z\left(z^{2}-3 / 4\right)\right)=0$. Let $z=\cos (u)=\left(e^{i u}+e^{-i u}\right) / 2$. Then $4 z\left(z^{2}-3 / 4\right)=\cos (3 u)=\cos 3 x \cosh 3 y+i \sin 3 x \sinh 3 y$. Thus we seek those $u$ for which $\operatorname{Re}\left(e^{3 i u}\right)=0$. Hence $e^{i u}=r e^{i \theta}$ where $\theta= \pm \pi / 6, \pm \pi / 2$, $\pm 5 \pi / 6, r>0$. Since $z=\cos \theta(r+1 / r) / 2+i \sin \theta(r-1 / r) / 2 \in Q$, we may write $z=\sqrt{3}(r+1 / r) / 4+i(r-1 / r) / 4$ where $0<r<1$. If we write $z=x+i y$ then $4 x^{2} / 3-4 y^{2}=1$ and $y<0$. Finally we compute the image of this 
half-hyperbola under the map $g_{3}^{-1}=(1-z) /(1+z)$. In terms of $r$ it is

$$
\frac{4 r-\sqrt{3}\left(r^{2}+1\right)-i\left(r^{2}-1\right)}{4 r+\sqrt{3}\left(r^{2}+1\right)+i\left(r^{2}-1\right)} .
$$

Thus the optimal curve $\gamma_{0}$ begins at 1 , follows the unit circle clockwise to the point -1 , then enters the unit disk $D$, making an angle of $\pi / 6$ with the positive $x$-axis, continues in the upper half disk $D^{+}$, until it meets $\mathbf{R}$ at right angles at the point $7-4 \sqrt{3} \cong .0718$.

\section{ON FUCHS' PROBLEM}

In this section, we show that if we remove the hypothesis that $\gamma$ is connected, the constant $c_{0}$ is no longer the lower bound for the harmonic measure at the origin. The proof below is motivated by the example in [7].

Suppose $E$ is a Borel subset of the closure of the right half-plane, $\bar{R}$, and suppose $E$ contains a free boundary arc on the imaginary axis. In other words, there exists a open disk $D(i a, r)$ with center at $i a, a$ real, and radius $r>0$ so that $D(i a, r) \cap E=\{i t: a-r<t<a+r\}$. If $0<\delta<r$, let $l_{1}$ denote the line segment from $i(a+\delta)$ to $\delta+i a$, let $l_{2}$ denote a line segment from $i(a+\delta / 2)$ to $i(a+\delta)$, and let $E_{\delta}=E \cup l_{1} \backslash l_{2}$. In the case when $E$ is the imaginary axis, $I$, and $a=0$, we denote this set by $I_{\delta}$. The idea of the argument below is that $w(z, I, R) \equiv 1$ and for $z$ fixed $w\left(\delta z, I_{\delta}, R\right)=c<1$, where $c$ is independent of $\delta$. So for an arbitrary set $E$, at a point in $R$ near $i a$, with $\delta$ extremely small, $E$ "looks like" $I+i a$ and $E_{\delta}$ "looks like" $I_{\delta}+i a$, and thus $w\left(z, E_{\delta}, R \backslash E_{\delta}\right)$ should be less than $w(z, E, R \backslash E)$.

Proposition. For each $z \in R \backslash E$, there is a $\delta_{0}>0$ so that $w\left(z, E_{\delta}, R \backslash E_{\delta}\right)<$ $w(z, E, R \backslash E)$ when $\delta<\delta_{0}$.

Proof. Without loss of generality, $a=0$. By the maximum principle, it suffices to show the inequality on $R \cap\{z:|z|=3 \delta\}$. Let $\sigma_{\varepsilon}=\left\{e^{i \theta}:\left|e^{2 i \theta}+1\right|<\right.$ $\varepsilon,-\pi / 2 \leq \theta \leq \pi / 2\}$ and let $\sigma_{0}=\left\{e^{i \theta}:\left|e^{i \theta}-1\right|<1 / 2\right\}$. Recall that $I_{1}$ is the set formed from the imaginary axis with $a=0$ and $\delta=1$, by the process described above. Let

$$
\varepsilon_{1}=\min _{e^{i \theta} \in \sigma_{0}}\left(1-w\left(2 e^{i \theta}, I_{1}, R \backslash I_{1}\right)\right) .
$$

By using an explicit conformal map of $R \backslash D(0,2)$ onto $R$, it is not hard to show that

$$
w\left(z, 2 \sigma_{\varepsilon}, R \backslash D(0,2)\right)<\frac{1}{3} \varepsilon_{1} w\left(z, 2 \sigma_{0}, R \backslash D(0,2)\right)
$$

when $|z|=3$, and $\varepsilon$ is sufficiently small.

Now for $z=2 \delta e^{i \theta}$ with $\theta$ fixed, $w(z, E, R \backslash E) \rightarrow 1$ as $\delta \rightarrow 0$ and $w\left(z, E_{\delta}, R \backslash E_{\delta}\right) \rightarrow w\left(2 e^{i \theta}, I_{1}, R \backslash I_{1}\right)<1$. Thus we may choose $\delta>0$ so that when $z=2 \delta e^{i \theta}$ with $e^{i \theta} \in \sigma_{0}$,

$$
w(z, E, R \backslash E)-w\left(z, E_{\delta}, R \backslash E_{\delta}\right)>\frac{1}{2} \varepsilon_{1}
$$


and so that when $z=2 \delta e^{i \theta}$ with $e^{i \theta} \in \partial D(0,1) \backslash \sigma_{\varepsilon}$

$$
w(z, E, R \backslash E)-w\left(z, E_{\delta}, R \backslash E_{\delta}\right)>0 .
$$

Thus for $|z|=2 \delta$

$$
\begin{aligned}
& w(z, E, R \backslash E)-w\left(z, E_{\delta}, R \backslash E_{\delta}\right) \\
& \quad \geq-w\left(z, 2 \delta \sigma_{\varepsilon}, R \backslash D(0,2 \delta)\right)+\frac{1}{2} \varepsilon_{1} w\left(z, 2 \delta \sigma_{0}, R \backslash D(0,2 \delta)\right) .
\end{aligned}
$$

We conclude that for $|z|=3 \delta$, by (4) and the above,

$$
w(z, E, R \backslash E)-w\left(z, E_{\delta}, R \backslash E_{\delta}\right) \geq\left(\frac{1}{2} \varepsilon_{1}-\frac{1}{3} \varepsilon_{1}\right) w\left(z, 2 \delta \sigma_{0}, R \backslash D(0,2 \delta)\right)>0 .
$$

This proves the proposition.

An easy consequence of this proposition is the following result of Hayman [7]. If $E$ is a Borel subset of the right half-plane $R$, let $E^{*}=\{i y: y>0$ and $e^{i \theta} y \in E$ for some $\left.\theta\right\}$.

Corollary 1. There is a set $F$ with $F^{*}=\{i y: y \geq 0\}$ and $w\left(1, F^{*}, R\right)=\frac{1}{2}>$ $w(1, F, R)$.

To see this, apply the proposition with $F=\left\{I_{\delta}+i\right\} \backslash\{i y: y<0\}$ for $\delta$ sufficiently small. We remark that this example doesn't really depend on the exact nature of the set $I_{\delta}$ near 0 .

If we let $E$ denote the image in $R$ of the extremal curve given in the previous section, under the linear fractional transformation $(1+z) /(1-z)$, and then apply the proposition, we obtain:

Corollary 2. If we do not assume that the set $\gamma$ in the statement of Theorem 1 is connected, then the harmonic measure of $\gamma$ at the origin can be smaller than $c_{0}$.

\section{REFERENCES}

1. Lars V. Ahlfors, Conformal invariants, McGraw-Hill, New York, 1973.

2. K. F. Barth, D. A. Brannan and W. K. Hayman, Research problems in complex analysis, Bull. London Math. Soc. 16 (1984), 490-517.

3. A. Beurling, Études sur un problème de majoration, Thèse, Uppsala, 1933.

4. T. Carleman, Sur les fonctions inverses des fonctions entieres, Ark. Mat. Astronom. Fys. 15 (1921).

5. C. FitzGerald, B. Rodin and S. Warschawski, Estimates of the harmonic measure of a continuum in the unit disk, Trans. Amer. Math. Soc. 287 (1985), 681-685.

6. J. B. Garnett, Applications of harmonic measure, Univ. of Arkansas Lecture Notes, vol. 8, Wiley-Interscience, New York, 1968.

7. W. Hayman, On a theorem of Tord Hall, Duke Math. J. 41 (1974), 25-26.

8. J. Jenkins, On a problem concerning harmonic measure, Math. Z. 135 (1974), 279-283.

9. Shizuo Kakutani, Two dimensional Brownian motion and harmonic functions, Proc. Imp. Acad. Tokyo 20 (1944), 706-714.

10. B. J. Maitland, A note on functions regular and bounded in the unit circle and small at a set of points near the circumference of the circle, Proc. Cambridge Philos. Soc. 35 (1939), 382-388. 
11. D. Marshall and J. Morrow, Unzipping plane domains (numerical computations of conformal maps), preprint.

12. H. Milloux, Le théorème de M. Picard, suites de fonctions holomorphes, fonctions méromorphes et fonctions entierès, J. Math. 3 (1924), 345-401.

13. __ Sur le théorème de Picard, Bull. Soc. Math. France 53 (1925), 181-207.

14. R. Nevanlinna, Über eine Minimumaufgabe in der Theorie der konformen Abbildung, Göttinger Nachr. I.37 (1933), 103-115.

15. L. N. Trefethen, Conformal map of a rectangle, informal notes, 1983.

16. _ Analysis and design of polygonal resistors by conformal mapping, Z. Angew. Math. Phys. 35 (1984), 692-704.

Department of Mathematics, University of Washington, Seattle, Washington 98195

Department of Mathematics, University of Tennessee, Knoxville, Tennessee 37916 